\title{
Robot Software Platform for IoT-Based Context-Awareness
}

\author{
Yongseong Cho*, Jongsun Choi ${ }^{\dagger}$ and Jaeyoung Choi \\ School of Computer Science and Engineering, \\ Soongsil University, 369 Sangdo-Ro, Dongjak-Gu, \\ Seoul 06978, Korea \\ *yongseong.cho@ssu.ac.kr \\ †jongsun.choi@ssu.ac.kr \\ †choi@ssu.ac.kr \\ Young-Jae Ryoo \\ Department of Control Engineering and Robotics, \\ Mokpo National University, 1666 Youngsan-Ro, \\ Cheonggye-Myeon, Muan-Gun, Jeonnam 58554, Korea \\ yjryoo@mokpo.ac.kr
}

Received 14 May 2016

Accepted 26 January 2017

Published 17 April 2017

\begin{abstract}
As Internet of Things (IoT)-based sensors have emerged recently, new technologies are being developed for users to provide suitable services by recognizing the surrounding situations. In the service robot sector, many researches are being carried out in order to exploit the collected data from IoT-based sensors. In addition, researches on robot middleware using context information are actively performed.

In this paper, we propose a robot SW platform, which provides IoT-based context-aware services. This robot software platform consists of Ontology Agent, Robot Service Execution Engine, and Context Handler. The Ontology Agent is a module that searches information, such as sensor location, value type, etc., of the IoT devices stored in Ontology Repository and provides them to the Context Handler. The Context Handler reads IoT devices' data and checks whether service transition conditions are met. If the conditions are satisfied, the data is delivered to the Robot Service Execution Engine, which is in charge of executing services. In experiments, we show a procedure executing the context-aware robot services through definition of robot services, a robot service execution engine, and a context handler, etc.
\end{abstract}

Keywords: Service robot; IoT (Internet of Things); context-awareness; robot software platform.

$\$$ Corresponding author.

This is an Open Access article published by World Scientific Publishing Company. It is distributed under the terms of the Creative Commons Attribution 4.0 (CC-BY) License. Further distribution of this work is permitted, provided the original work is properly cited. 


\section{Introduction}

As the Internet of Things (IoT) technology has emerged, sensors are being newly defined. The IoT technology can connect all the things that surround us with the Internet by integrating sensor and communication functions and can help provide intelligent services by exploiting such sensors. ${ }^{1-5}$ Because IoT-based sensors are equipped with their own data processing and communication functions, they can easily support context-aware services.

The context-aware technology exploiting IoT-based sensors affects the robot development ${ }^{6-8}$ as well. The recent robot industry has expanded from existing industrial robots to service robots. Consequently, various types of robots supporting humans' daily life, like humanoid robots and nursing robots are emerging. ${ }^{9-11}$ Unlike existing industrial robots, such service robots must recognize surrounding situations and provide appropriate services. To this end, methods are studied to handle context-awareness surrounding users with IoT-based sensors. ${ }^{6-8,12}$

Industrial robots were usually developed according to the conventional software development methodology of developing codes to control hardware, compiling and executing the codes. The industrial robots can be easily developed with such a development methodology because they do not have to consider the surrounding environment. However, unlike industrial robots, it is difficult to develop service robots only with codes to control robot hardware. To provide desired services by recognizing the surrounding environments, hardware control of the robot and sensor information on service environment should be combined. For this reason, requirements for processing context-awareness are increasing in the recent robot industry, and researches for combining context-awareness with robot platforms and sensors are being actively researched. ${ }^{13-15}$

Most researches, however, carry out simply by adding a module to the existing robot platform and/or middleware to provide context-aware services. Although such methods ${ }^{16,17}$ might be efficient in a simple, specific application domain, it is difficult to apply the method to the IoT environments with a variety of heterogeneous data types of various sensors.

In this paper, we propose a robot SW platform to provide IoT-based contextaware services, which uses RDF-based Ontology Repository to apply IoT devices to context-aware services. IoT devices' information such as location, value type, etc. IoT devices are defined in the Ontology Repository and used to process contextawareness. The context-aware processing engine of the proposed robot SW platform reads the data of IoT devices, based on the predefined information of these devices, and uses this information to process context-awareness.

This paper is organized as follows. Section 2 examines the related works on robot development platforms, middleware, and context-aware technologies. Section 3 explains the overview of the robot SW platform and its components. Section 4 shows the process of context-awareness and process of executing context-aware robot services. Section 5 includes an experiment of the process to execute IoT-based 
context-aware services on the proposed robot SW platform. Lastly, Sec. 6 provides the conclusion of the paper.

\section{Related Work}

This section introduces key robot development platforms and middleware. We briefly examine methods for providing context-aware services based on ontology and investigate other works for developing service robots. Lastly, we explain context-aware workflow language, CAWL, which is used as execution files on the proposed robot SW platform.

\subsection{Robot platform}

Robot Operating System (ROS) $)^{18,19}$ is an open source operating system developed in the US. Like general operating systems, ROS provides hardware abstraction, low level device control, and API. ROS is currently the most popular operating system used for developing robots. Open Platform for Robotic Services (OPRoS ${ }^{20}$ is a component-based robot middleware developed in Korea. OPRoS assures reusability, portability, and extendibility by providing the functions for supporting existing architectures. OPRoS provides robot developers with higher convenience by supporting various development tools such as debugger, simulator, etc., compared to other platforms and middleware. Robotics Technology middleware RT-middleware ${ }^{21}$ is a platform developed in Japan, through which users first develop software to control each device, then, combine them and use them hierarchically. RT-middleware looks similar to OPRoS, but it supports development tools insufficiently.

As the previously mentioned platforms and middleware mainly focus on the implementation of robot's hardware functions, robot developers can easily adapt robot specifications and develop robot functions; however, it is not easy to control robots using external devices such as IoT-based sensors and Human Robot Interface (HRI) modules because, in general, interfaces or software modules for each sensors are needed in case of moving or changing devices, and robot developers should know how to manage them. Meanwhile, Remote Open Campus System (ROCS), ${ }^{22}$ being developed in Japan, is a system that provides services in which a user can control robot's location in a remote place. As a robot system helps a user to walk around campus virtually, ROCS can support image-processing functions with robot's vision module. Robot Service initiative (RSi)-Cloud ${ }^{23}$ is a system that utilizes Robot Service Network Protocol (RSNP) to execute predefined robot services through a robot in a remote place. It has advantages in which robot services can be easily reused and robots in RSi-Cloud can be easily managed with Web-based monitoring. In contrast, the suggested software platform is able to offer various context-aware robot services to end-users using context information based on sensor data, which is obtained from various IoT devices and user profiles. 


\subsection{Context-awareness based on Ontology definitions}

In order to process context-awareness using the values extracted from sensors, systems, data structures, and data semantics of various sensors are needed to be matched with each other. Otherwise, it becomes difficult to use the sensor data to process context-awareness because of heterogeneity of each sensor. In order to solve this problem, there are variety of works exploiting ontology-based context information. ${ }^{24,25}$ They describe the information of sensors on ontology using Resource Description Framework (RDF), RDF Schema (RDFs), and Web Ontology Language (OWL). The described information is used to store and extract sensor values, and it helps to recognize current context by ontology inference engine. Context-Aware Workflow Language $(\mathrm{CAWL})^{26}$ refers to a context-aware workflow language to be able to describe situational information on user's surroundings. CAWL can compose of a workflow based on Web services, and can describe context-aware information as the transition condition of executing robot services. The transition conditions use the keyword described in the Ontology Repository. Context-aware conditions can be defined more intuitively by describing them in the Subject-Verb-Object (SVO) format. We showed an experiment in Sec. 5, in which CAWL document is used to develop a robot service using a bio signal as a context-aware transition condition.

\section{Context-Aware Robot SW Platform}

This section introduces the overview and structure of robot SW platform, which can provide IoT-based context-awareness, and explains each component. Figure 1 shows the diagram of robot SW platform that can offer IoT-based context-awareness.

The proposed robot SW platform uses Ontology Repository in Fig. 1, which includes context information, in order to describe robot services on the basis of context-awareness. Ontology Repository stores keywords and their definitions, which are required for context-aware expression. These keywords have defined a domain for robot services to be used, and they can be searched or gathered from the Ontology Repository. Robot Service Execution Engine is a component to execute CAWL documents. The engine receives the help of the Context Handler to control a robot by analyzing robot services defined in the CAWL document and recognizes the context surrounding the end-user.

The Context Handler is a component to process context-awareness that is required for executing robot services. It is used for processing context-awareness by checking the information of IoT devices defined in the Ontology Repository and by reading the values of IoT devices in the actual execution environment. The Context Handler works together with the Robot Service Execution Engine in an organized manner and it recognizes the context surrounding the end user to execute robot services.

Figure 2 shows components of the robot SW platform, which provides IoT-based context-aware functions. The robot SW platform consists of the Ontology Agent, Robot Service Execution Engine, and Context Handler. 


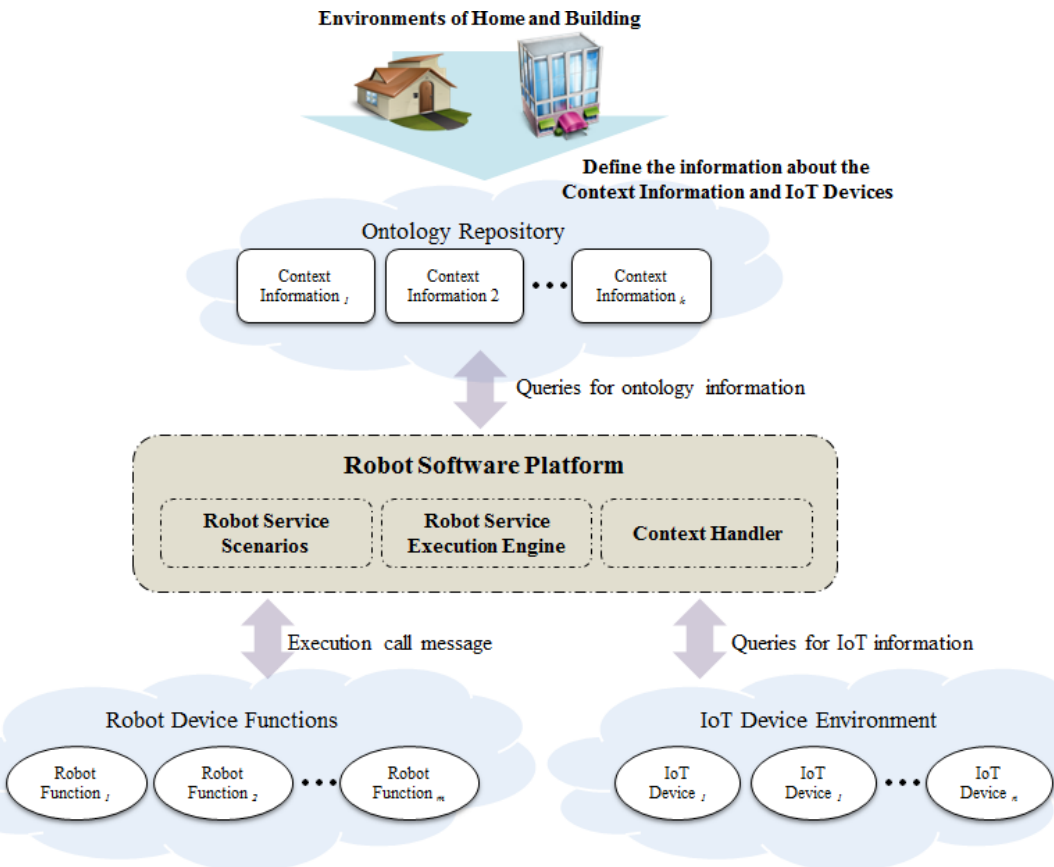

Fig. 1. Suggested robot SW platform overview.

\subsection{Ontology agent}

The Ontology Agent is a module that can read and search the values defined in the Ontology Repository, where keywords are stored to define robot services. These keywords are used for processing context-awareness, and each keyword contains keyword name, IP address, port number, and data type. All the keywords defined in the Ontology Repository are used for defining robot services. The Robot Service Execution and the Context Handler access keywords through the Ontology Agent.

\subsection{Robot service execution engine}

The Robot Service Execution Engine is a module for executing robot services defined in the CAWL document. It extracts nodes to be executed and their transition conditions by analyzing the context-aware workflow in detail. The extracted execution nodes and their transition conditions are arranged in order of execution. Each node is executed if its transition conditions are satisfied. The transition conditions are processed by the Context Handler, and the Robot Service Execution Engine executes the node if the Context Handler delivers "True".

\subsection{Context handler}

The Context Handler is a module to judge the context information for executing standby state. After the Robot Service Execution Engine extracts the transition 


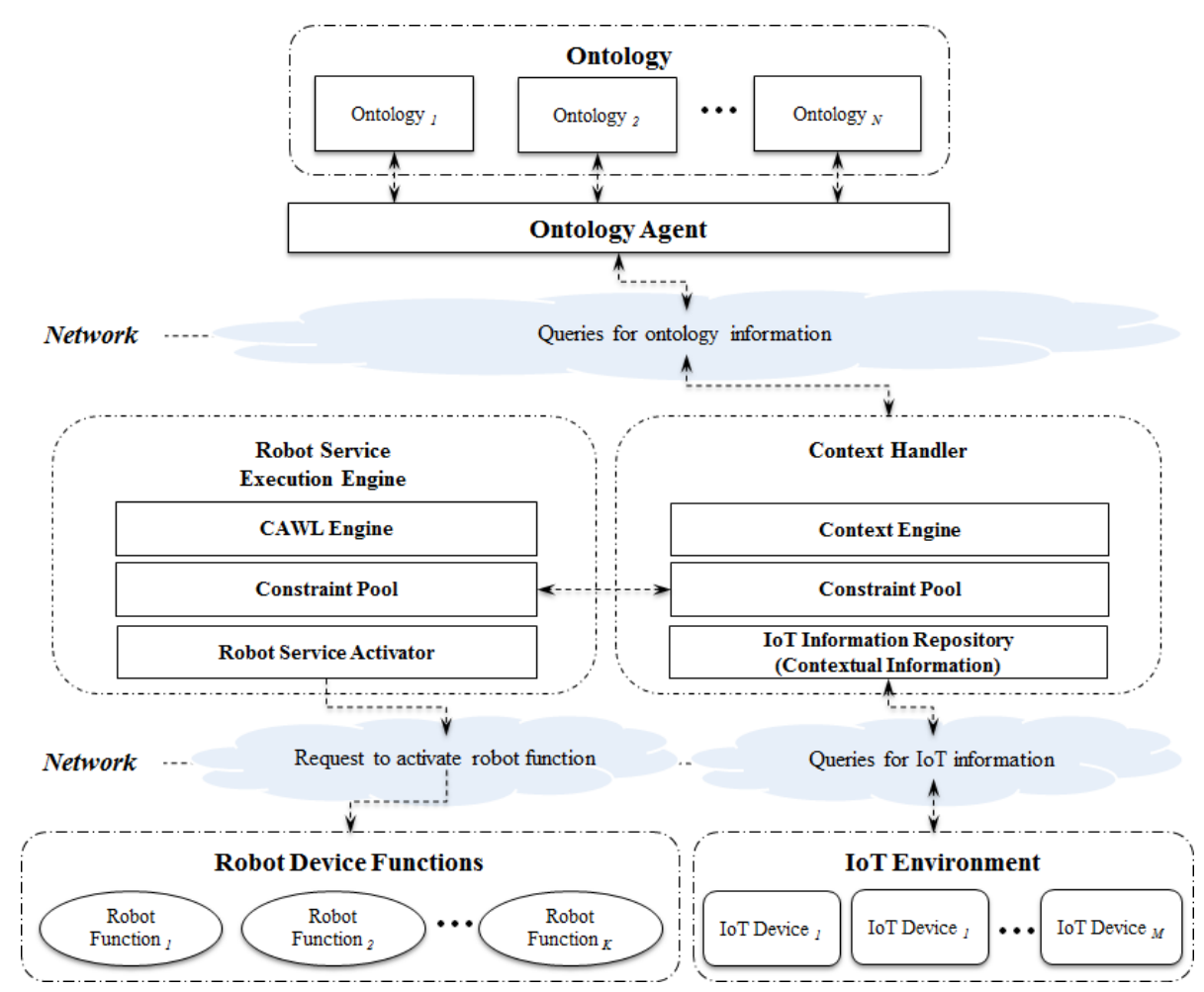

Fig. 2. Suggested robot SW platform architecture.

conditions of each executing node, defined in the CAWL document, they are delivered to the Context Handler along with the ID of each transition conditions. All transition conditions delivered from the Robot Service Execution Engine are changed to the IoT device information, defined in the Ontology Repository through the Ontology Agent. The Robot Service Execution Engine delivers IDs of the transition conditions of the standby execution states to the Context Handler, if a specific node's executing order arrives. The Context Handler monitors the IoT devices of the IDs concerned and delivers transition conditions to the Robot Service Execution Engine, if they are judged to be true. If they are not true, they are continuously checked.

\section{Context-Aware Process in Robot SW Platform}

This section deals with a process of processing IoT-based context-awareness and a process for executing robot services.

Figure 3 shows the context-aware process in the proposed robot SW platform. The Robot Service Execution Engine receives the CAWL document, where robot services are defined, and extracts information required for executing robot services. In this process, context-aware conditions to execute each node can be identified. 


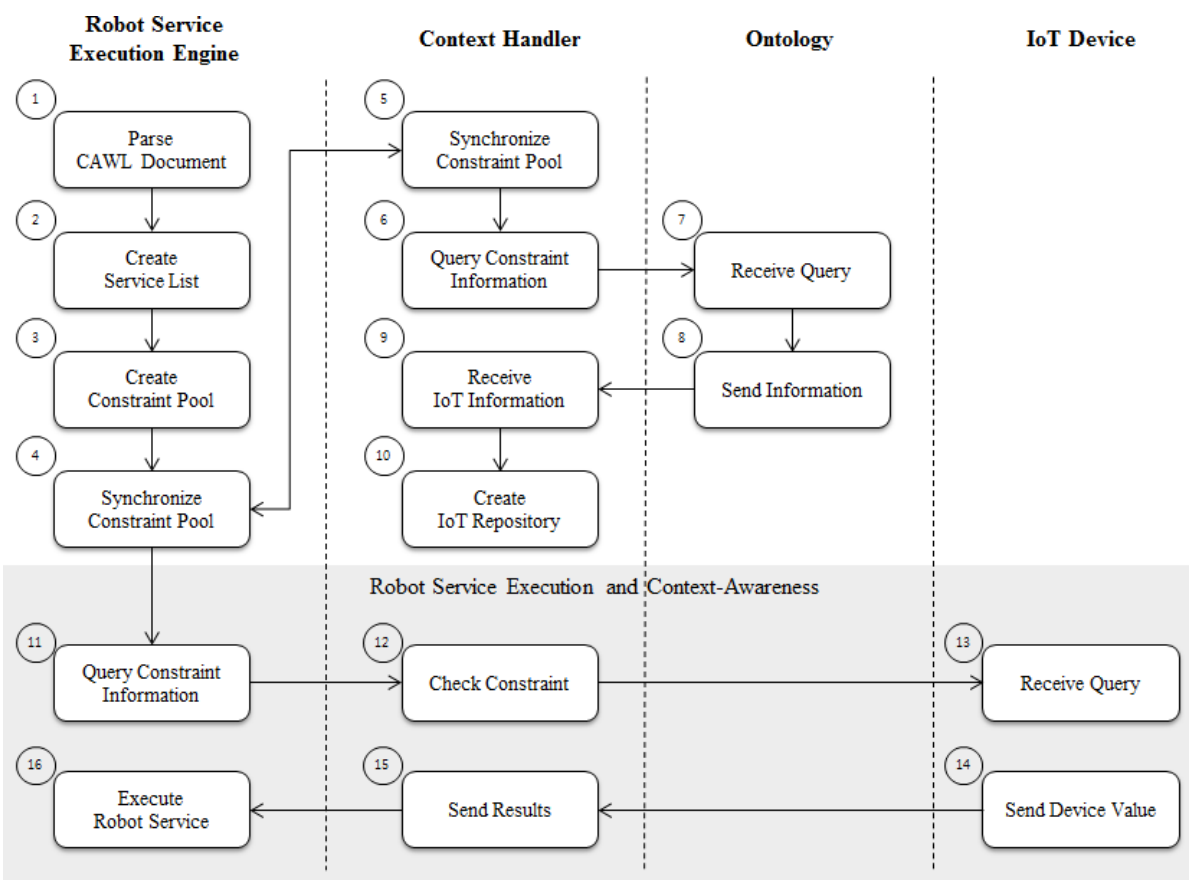

Fig. 3. Context-awareness procedure in the IoT environment.

Table 1. Constraint pool information.

\begin{tabular}{cl}
\hline ID & \multicolumn{1}{c}{ Indexes of contextual constraint } \\
\hline Subject & Subject keyword for contextual constraint \\
& Usage $\rightarrow$ \{Ontology Class $\} /$ Subject $\}$ \\
Verb & Verb keyword for contextual constraint \\
& Usage $\rightarrow\{$ Verb $\}$ \\
Object & Object keyword for contextual constraint \\
& Usage $\rightarrow\{$ Ontology Class $\} /$ OObject $\}$ \\
\hline
\end{tabular}

The Robot Service Execution Engine registers the context-aware conditions in Constraint Pool, in terms of the information defined in Table 1.

The Robot Service Execution Engine sends all information registered in Constraint Pool to the Context Handler before the robot services are executed. The Context Handler stores such information in its own Constraint Pool in the same format as the Robot Service Execution Engine. The Context Handler creates additional memory spaces in the IoT Information Repository in this process. The details of the IoT Information Repository can be seen in Table 2.

The IoT Information Repository stores core information required for processing context-awareness. Its creation process is that first, the Context Handler contacts the Ontology Agent by using the information of context conditions delivered from 
Table 2. Information entries in the IoT information repository.

\begin{tabular}{ll}
\hline C_ID & \multicolumn{1}{c}{ Constraint ID } \\
\hline S_ID & A unique ID for A Sensor and IoT device \\
Name & A human-friendly distinguishable Keyword for A Sensor and IoT device \\
IP:PORT & Target Sensor's IP and Port number \\
Expr. Func. & An intermediate function to convert sensor raw values to human-friendly \\
& $\quad$ proper values \\
Basis Value & A base sensor value to decide true or false for the surrounding environment \\
Current Value & A current sensor and IoT device value \\
Status & A current state of target sensor and IoT device \\
\hline
\end{tabular}

Table 3. Example of contextual constraints.

\begin{tabular}{cccc}
\hline ID & Subject & Verb & Object \\
\hline 0 & Tom & isLocatedIn & Room307 \\
\hline
\end{tabular}

the Robot Service Execution Engine. All context conditions have the SVO form, where $\mathrm{S}$ and $\mathrm{O}$ are mainly the comparison subjects, and $\mathrm{V}$ represents IoT module function. For example, as defined in Table 3, three keywords are used as "Tom", "isLocatedIn", and "Room307".

When those keywords are searched in the Ontology Agent, the information can be revealed in Tables 4 and 5. When the subject Tom is checked, it is the individual,

Table 4. Information of constraints keyword to the ontology.

\begin{tabular}{lll}
\hline Keyword & \multicolumn{1}{c}{ Data property name } & Data property value \\
\hline Tom & UserID & 100062 \\
& PersonName & Tom \\
& PersonNickName & Tommy \\
& PersonValidateFunction & Tag \\
& PersonFunctionValue & Tag100062 \\
& TargetSensorName & EntryServer \\
Room307 & OwnSensorName & R307 \\
& OwnSensorTargetIP & 192.168 .0 .8 \\
& OwnSensorTargetPort & 9062 \\
& OwnSensorFunctionBoolean & false \\
& OwnSensorFunction & V \\
& TargetSensorBasisValue & \% UserID\% \\
& TargetSensorFunctionType & EqualsTo \\
\hline
\end{tabular}

Table 5. Example of object property to the ontology.

\begin{tabular}{lcc}
\hline Object property & Domain & Range \\
\hline isLocatedIn & Person & Room \\
\hline
\end{tabular}


Table 6. Example of storing information related to IoT.

\begin{tabular}{ccccccc}
\hline C_ID & S_ID & Name & IP:PORT & Expr. func. & Basis value & Current value \\
\hline 0 & E001 & Tom & $192.168 .0 .7: 9061$ & $\mathrm{v}$ & 100062 & 100062 \\
\hline
\end{tabular}

defined in person class and has information such as ID, name, etc. When the object Room307 is checked, it is the individual, defined in room class. Actually, it confirms that OwnSensorName, which is a sensor name, and TargetSensorBasisValue, which is a value to compare with sensor's IP address, Port number, and sensor's value, are defined. Such information is important for processing context-awareness and is used in the Verb keywords.

Lastly, when a verb isLocatedIn is checked, it confirms that it is defined in object properties. Object properties on ontology offer a connection function between individuals. Also, the current isLocatedIn keyword provides a connection function between Tom in person class and Room307 in room class. Based on the information mentioned above, the Context Handler creates the IoT information Repository. The context information in Tables 4 and 5 is registered as IoT information in Table 6 .

The information registered in the IoT Information Repository works as an object. The IoT information object periodically reads values from a sensor that the object concerned should refer to and judges the current situation using the condition information of the sensor concerned. The IoT information object in Table 5 periodically reads the value from the sensor installed in Room307, according to the connection relationship defined in the isLocatedIn keyword, and checks whether the value is the same as the ID of Tom. If it is, the Context Handler recognizes that Tom has currently entered Room307.

"Robot Service Execution and Context-Awareness" in Fig. 3 shows the process of executing robot services using the result of processing context-awareness. The Robot Service Execution Engine delivers all information in the Constraint Pool to the Context Handler. Also, the engine delivers the context condition ID of the current standby state to the Context Handler. The Context Handler checks the IoT information connected with the context condition concerned and makes the object concerned to operate. The IoT information object executes the context-awareness that the object concerned should process, and then it records the result in the Constraint Pool. The Context Handler periodically checks the result and returns the result of the context condition to the Robot Service Execution Engine. The Robot Service Execution Engine executes the node, if the context condition of the current standby state becomes true, and delivers the context condition ID of the next node to the Context Handler. Such a process is repeated until all nodes are executed.

\section{Implementation and Experiments}

In this section, we show the process of how to execute robot SW platform using IoT device which offers temperature data. Proposed robot SW platform can recognize the 


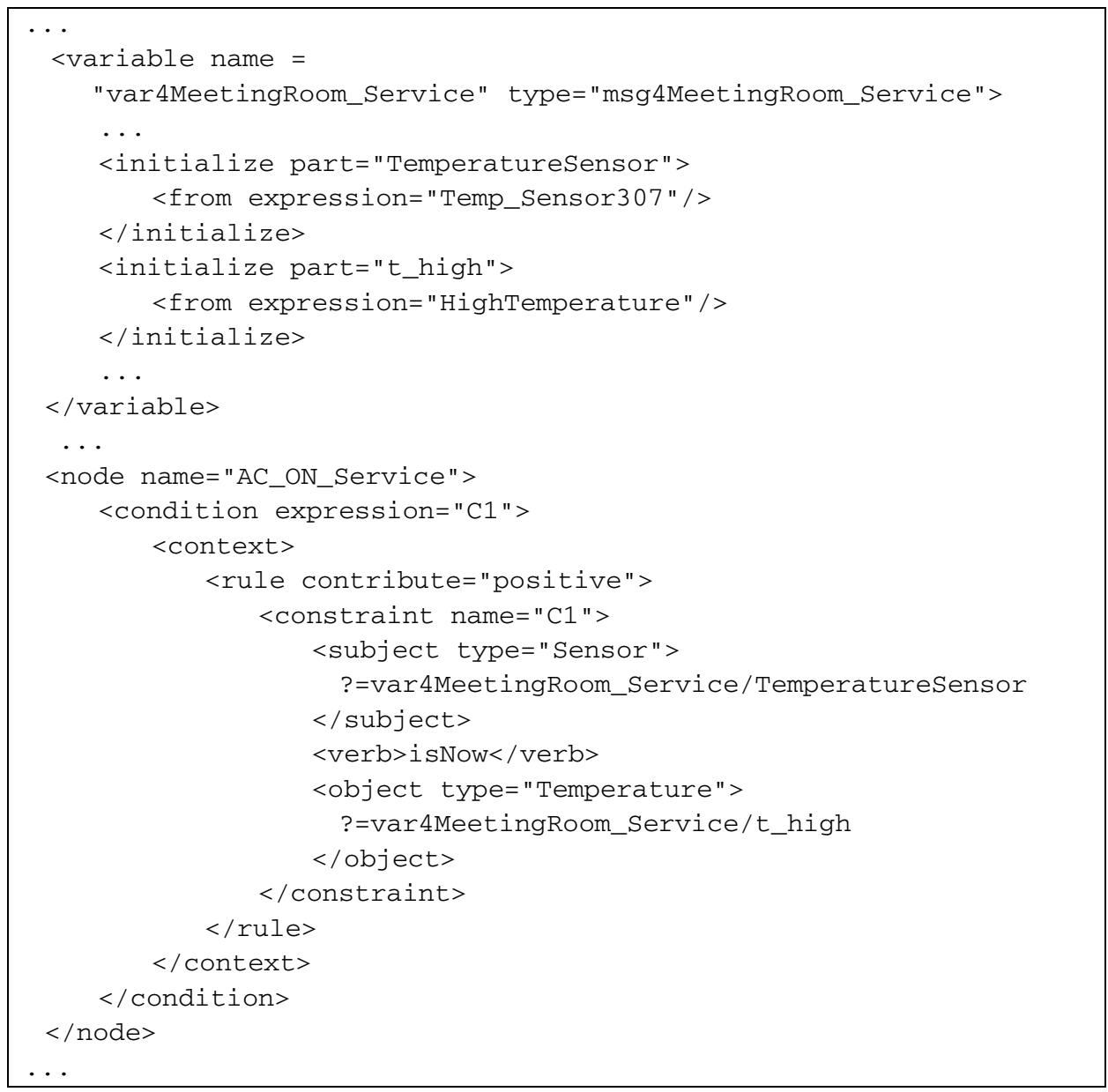

Fig. 4. Robot service definition for using IoT devices.

cases, for example, temperature data, which is offered by IoT device is changed to more than a specific level, and execute pre-defined robot service.

\subsection{Definition of robot services}

Figure 4 is a part of the CAWL document, where a robot service is defined for the experiment. A service node defined in Fig. 4 is AC_ON_Service, and its context-aware conditions are defined as Temp_Sensor307(S), isNow(V), and HighTemperature(O). The keywords defined in the context-aware conditions are explained in Sec. 5.2. The experiment in Sec. 5.5 shows the process that the context-aware conditions are recognized and finally the AC_ON_Service service node is executed. 
Table 7. One of individual examples to the ontology for collecting temperature information.

\begin{tabular}{lll}
\hline Keyword & \multicolumn{1}{c}{ Data property name } & Data property value \\
\hline Temp_Sensor307 & OwnSensorName & s001 \\
& OwnSensorTargetIP & 192.168 .11 .11 \\
& OwnSensorTargetPort & 9063 \\
& OwnSensorTargetFunctionBoolean & true \\
& OwnSensorFunction & (v-0.5)*100.0 \\
HighTemperature & TargetSensorName & Temp_Sensor \\
& TargetSensorFunctionType & Greaterthan \\
& TargetSensorBasisValue & 25.0 \\
\hline
\end{tabular}

Table 8. Example of object property to process temperature-based context-awareness.

\begin{tabular}{lcc}
\hline Object property & Domain & Range \\
\hline isNow & Sensor & Temperature \\
\hline
\end{tabular}

\subsection{Definition of robot services}

Tables 7 and 8 show that the information of the IoT devices is defined in ontology for processing context-awareness. Table 7 shows the subject and object keywords used as context-aware conditions in the CAWL document. Temp_Sensor307 is the keyword used as a subject, and it contains information such as name, IP, Port, etc. of the IoT device used for context-awareness. HighTemperature is the keyword used as an object, and it contains information to compare the data of IoT device defined in the Temp_ Sensor307 keyword.

Table 8 shows the verb keyword to be used as context-aware conditions in the CAWL document. isNow keyword defines constraint conditions to use the subject and object identified in Table 7. The sensor class, where Temp_Sensor307 keyword belongs, is designated as domain, and the Temperature class, where HighTemperature keyword belongs, is set as range.

\subsection{Robot service execution engine}

Figure 5 shows the execution of the CAWL document in Sec. 5.2 through the Robot Service Execution Engine.

Figure 5 (A) shows AC_ON_Service in standby state. The service nodes in standby state are emphasized as in Fig. 5. The context-aware conditions of AC_ON_Service are shown in Fig 5 (B). Temp_Sensor307(S), isNow(V), and HighTemperature $(\mathrm{O})$ appear in order. Figure 5 (C) shows a log message that AC_ON_Service node is in standby state for execution. AC_ON_Service is in standby state until the Context Handler recognizes context and returns the results. 


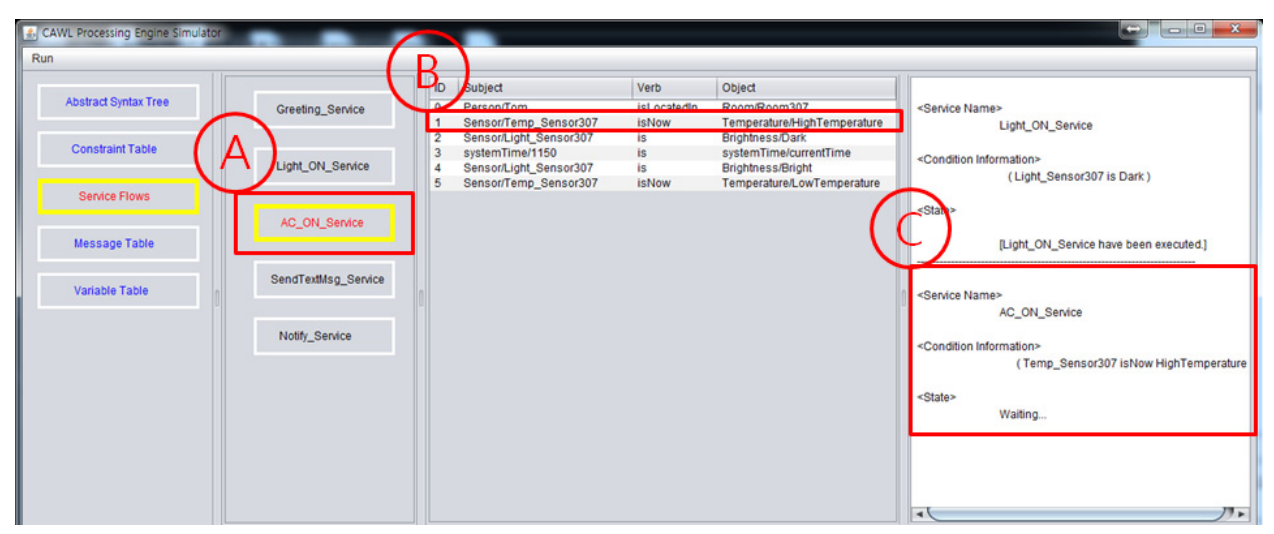

Fig. 5. A snapshot of Robot Service Execution engine.

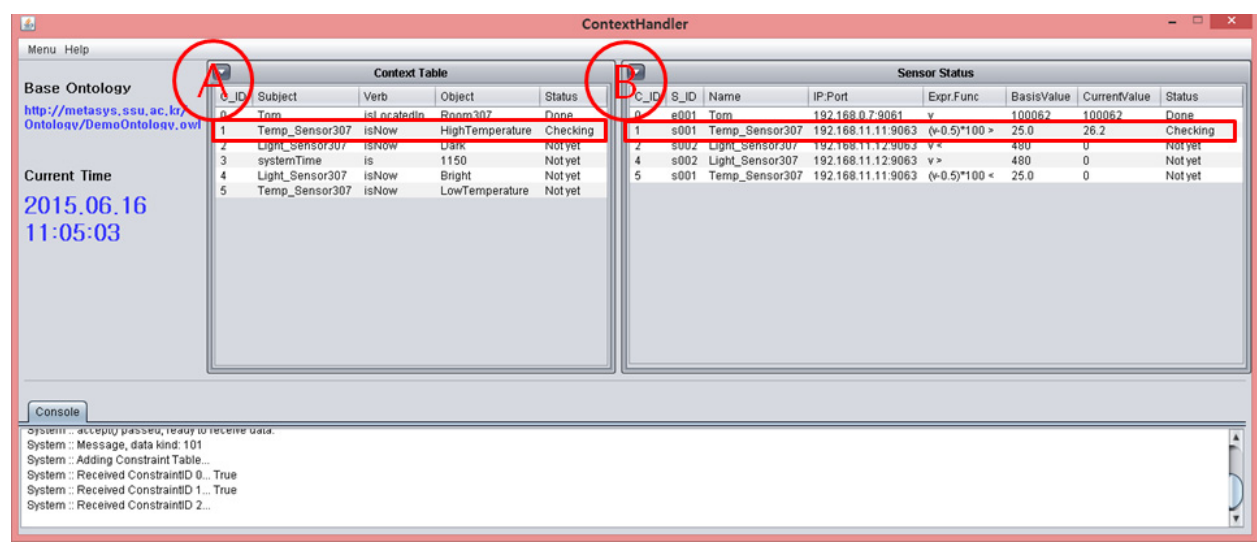

Fig. 6. A snapshot of Context Handler.

\subsection{Context handler}

Figure 6 shows the process of the Context Handler. In Fig. 6 (A), it can be confirmed that the context conditions checked in Fig. 5 (B) are registered. The context conditions in Fig. 5 (B) are converted to sensor status information as in Fig. 6 (B), and they use ontology information in Sec. 5.1.

Context Handler recognizes surrounded situation based on the information, which is displayed on B in Fig. 6. Activated sensors for executing context-aware system are set as "Checking" on the element Status. If it recognizes some situational change, it sends the recognized values to robot service execution engine. And then, Context Handler collects data of IoT device, which can offer temperature data based on information that is checked on B in Fig. 6. Context Handler compares the data with BasisValues, which can also be checked on B in Fig. 6, and executes defined contextaware service. At this time, activated IoT device for data collection and analysis is 


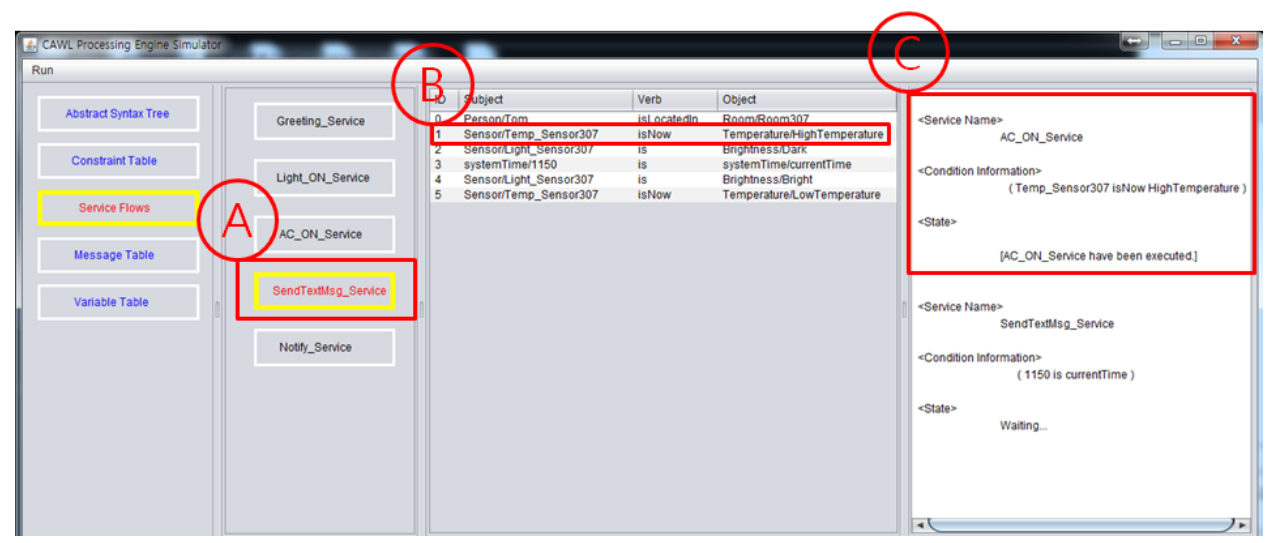

Fig. 7. Robot Service Execution triggered by Robot Service Execution Engine (Debug mode).

displayed as "Checking" on element Status. If it recognizes some contextual change, it will send the value to execution engine.

\subsection{Robot service execution process}

Figure 7 shows that Robot Service Execution Engine runs AC_ON_Service. Robot Service Execution Engine waits for one of service nodes invoked from Context Handler while sensor data or other circumstances are sufficient for among conditions of service nodes. When one service node invoked, Robot Service Execution Engine waits for following node's state to be invoked. Figure 7 (A) shows that the following node is in standby state for execution after AC_ON_SERVICE is executed. For the experiment, following node's service is about checking temperature information again, and generating a log message to confirm that AC_ON_SERVICE has been properly executed. (B) in Fig. 7 shows checking temperature information from one of IoT sensors, (C) in Fig. 7 shows the detailed message.

\section{Conclusion}

This paper has proposed a robot SW platform to provide IoT-based context-aware function. The proposed platform is designed to provide context-aware services by recognizing context surrounding end-users with various IoT-based sensors outside of a robot. The proposed platform consists of three main components: Ontology Agent searches information such as sensor location, value type, etc., of IoT device sensors stored in the Ontology Repository and provides the information to the Context Handler; Robot Service Execution Engine executes robot services based on robotservice-definition document; and Context Handler processes the service transition conditions described in the robot-service-definition document and delivers the result to the Robot Service Execution Engine. 
With the experiment, we showed that it is possible to provide context-aware robot services to end-users with IoT-based sensors on the proposed platform. And we showed that the sensor data are converted to context information, which is convenient for providing context-aware services. We expect that the method for processing context-awareness in the proposed robot SW platform would be very helpful for other service robots. In the near future, we are going to improve the robot SW platform and to apply it to various robots.

\section{Acknowledgments}

This research was supported by Basic Science Research Program through the National Research Foundation of Korea (NRF) funded by the Ministry of Science, ICT and Future Planning (No. 2016R1C1B2009744), and was supported by the Industrial Convergence Core Technology Development Program (No. 10048474) funded by the Ministry of Trade, Industry and Energy (MOTIE), Korea.

\section{References}

1. Q. Zhu, R. Wang, Q. Chen, Y. Liu and W. Qin, IOT gateway: Bridging wireless sensor networks into internet of things, 2010 IEEE/IFIP 8th Int. Conf. Embedded and Ubiquitous Computing (EUC) (December 2010), pp. 347-352.

2. S. D. T. Kelly, N. K. Suryadevara, and S. C. Mukhopadhyay, Towards the implementation of IoT for environmental condition monitoring in homes, IEEE Sensors Journal, (May 2013), pp. 3846-3853.

3. C. Perera, A. Zaslavsky, P. Christen, M. Compton and D. Georgakopoulos, Contextaware sensor search, selection and ranking model for internet of things middleware, 2013 IEEE 14th Int. Conf. Mobile Data Management (MDM) (June 2013), pp. 314-322.

4. S. Li, L. D. Xu and X. Wang, Compressed sensing signal and data acquisition in wireless sensor networks and internet of things, IEEE Transactions on Industrial Informatics (February 2014), pp. 2177-2186.

5. Q. Chi, H. Yan, C. Zhang, Z. Pang and L. D. Xu, A reconfigurable smart sensor interface for industrial WSN in IoT environment, IEEE Transactions on Industrial Informatics (February 2014), pp. 1417-1425.

6. E. Spaho, A. Sula, K. Matsuo, L. Barolli, F. Xhafa and J. Iwashige, An integrated system of robot, smartbox and RFID as an approach for internet of things, 2013 Eighth Int. Conf. Broadband and Wireless Computing, Communication and Applications (BWCCA) (October 2013), pp. 589-594.

7. P. Brizzi, D. Conzon, H. Khaleel, R. Tomasi, C. Pastrone and A. M. Spirito, Bringing the internet of things along the manufacturing line: A case study in controlling industrial robot and monitoring energy consumption remotely, 2013 IEEE 18th Conf. Emerging Technologies and Factory Automation (ETFA) (September 2013), pp. 1-8.

8. M. Murar and S. Brad, Rapid development of control algorithms and interfaces for remote monitoring of robotic arm through internet of things (IoT), in New Trends in Mechanism and Machine Science, Vol. 24 (Springer, 2015), pp. 941-948.

9. B. C. da Silva, G. Baldassarre, G. Konidaris and A. Barto, Learning parametrized motor skills on a humanoid robot, 2014 IEEE Into Conf. Robotics and Automation (ICRA), (June 2014), pp. 5239-5244. 
10. K. Nishiwaki, J. Chestnutt and S. Kagami, Planning and control of a humanoid robot for navigation on uneven multi-scale terrain, in Experimental Robotics, Springer Tracts in Advanced Robotics, Vol. 79 (2014), pp. 401-415.

11. A. Ioannou, E. Andreou and M. Christofi, Pre-schoolers' interest and caring behaviour around a humanoid robot, Tech Trends 59(2) (2015) 23-26.

12. Z. Shi, J. Tu, Q. Zhang, L. Liu and J. Wei, A survey of swarm robotics system, advances in swarm intelligence, Lect. Notes Comput. Sci. 7331 (2012) 564-572.

13. S. Lee and P. Y. Oh, Sensor information analysis for a humanoid robot, Int. J. Control Autom. Syst. 13(1) (2015) 175-181.

14. A. Pennisi, F. Previtali, C. Gennari, D. D. Bloisi, L. Iocchi, F. Ficarola, A. Vitaletti and D. Nardi, Multi-robot surveillance through a distributed sensor network, in Cooperative Robots and Sensor Networks 2015, Studies in Computational Intelligence, Vol. 604 (2015), pp. 77-98.

15. C.-W. Hsiao, Y.-H. Chien and W.-Y. Wang, Wall following and continuously stair climbing systems for a tracked robot, 2015 IEEE 12th Int. Conf. Networking, Sensing and Control (ICNSC) (April 2015), pp. 371-375.

16. J. Choi, Y. Lee, Y.-J. Ryoo, J. Choi and J. Choi, Action petri net for specifying robot motions, Int. J. Hum. Robot. 11(4) (2014) 1-12.

17. Y.-J. Ryoo, Walking engine using ZMP criterion and feedback control for child-sized humanoid robot, Int. J. Hum. Robot. 13(4) (2016) 1650021.

18. L. Peppoloni, F. Brizzi, C. A. Avizzano and E. Ruffaldi, Immersive ROS-integrated framework for robot teleoperation, 2015 IEEE Symp. 3D User Interfaces (3DUI) (March 2015), pp. 177-178.

19. T. Pinho, A. P. Moreira and J. Boaventura-Cunha, Framework using ROS and simtwo simulator for realistic test of mobile robot controllers, in CONTROLO'2014 - Proc. 11th Portuguese Conf. Automatic Control, Lecture Notes in Electrical Engineering, Vol. 321 (2015), pp. 751-759.

20. C. Jang, B. Song, S. Jung and S. Kim, A heterogeneous coupling scheme of OPRoS component framework with ROS, 2012 9th Int. Conf. Ubiquitous Robots and Ambient Intelligence (URAI) (November 2012), pp. 298-301.

21. N. Ando, T. Suehiro, K. Kitagaki, T. Kotoku and W.-K. Yoon, RT-middleware: Distributed component middleware for RT (robot technology), 2005 IEEE/RSJ Int. Conf. Intelligent Robots and Systems, 2005 (IROS 2005) (August 2005), pp. 3933-3938.

22. T. Kusu, Y. Ito, T. Kida, T. Shimada, M. Takahashi, Y. Nomoto, Y. Tsuchiya, M. Narita and Y. Kato, A virtual campus tour service using remote control robots on robot service network protocol, 2013 27th Int. Conf. Advanced Information Networking and Applications Workshops (WAINA) (March 2013), pp. 959-964.

23. Y. Kato, T. Izui, Y. Tsuchiya, M. Narita, M. Ueki and Y. Murakawa, RSi-cloud for integrating robot services with internet services, IECON 2011 - 37th Annual Conf. IEEE Industrial Electronics Society (November 2011), pp. 2158-2163.

24. A. Chebba, T. Bouabana-Tebibel and S. H. Rubin, Context in ontology for knowledge representation, in Advanced Computational Methods for Knowledge Engineering, Advances in Intelligent Systems and Computing, Vol. 358 (2015), pp. 311-320.

25. M. Görner, T. Göschel, S. Kassel, S. Sander and T. Klein, An ontology for interoperability: Modeling of composite services in the smart home environment, in Enterprise Interoperability, Lecture Notes in Business Information Processing, Vol. 213 (2015), pp. $30-38$.

26. J. Choi, Y. Cho and J. Choi, The design of a context-aware workflow language for supporting multiple workflows (in Korean), J. Korean Soc. Internet Inform. 10(6) (2009) $145-157$. 


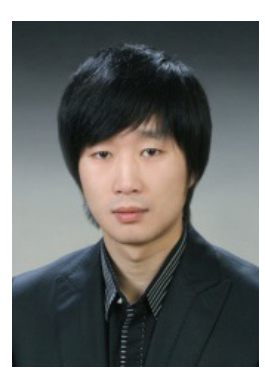

Yongseong Cho received the Master's degree in School of Computer Science and Engineering, Soongsil University, Seoul, Korea, in 2013. He was a Ph.D. candidate in School of Computer Science and Engineering, Soongsil University, Seoul, Korea.

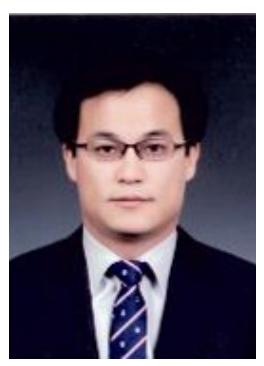

Jongsun Choi received the B.Sc. degree in School of Computer Science and Engineering from Soongsil University, Seoul Korea, in 2000 and the M.Sc. degree and Ph.D. degree in Department of Computer Graduate School from Soongsil University, Seoul, Korea, in 2002 and 2010 respectively. He has worked at Intelligent Robot Research Center of Soongsil University as a Research Professor (2010-2011) and at Seoil University as a Full-Time Lecture Professor (2012). He is currently an Assistant Professor of School of Computer Science and Engineering at Soongsil University, Seoul, Korea.

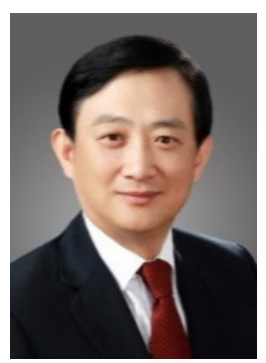

Jaeyoung Choi received the B.Sc. degree in Department of Control and Instrumentation Engineering, from Seoul National University, Seoul, Korea, in 1984, the M.Sc. degree in Department of Electrical Engineering, University of Southern California in 1986, and the Ph.D. degree in School of Electrical Engineering from Cornell University in 1991. He has previously worked at Oak Ridge National Laboratory (1992-1994) and University of Tennessee, Knoxville (1994-1995) as a postdoctoral Research Associate and a Research Assistant Professor, Respectively, where he had been involved with the ScaLAPACK project. He is currently a Professor of School of Computer Science and Engineering at Soongsil University, Seoul, Korea. His research interests include robot middleware, cloud computing and high-performance computing. 


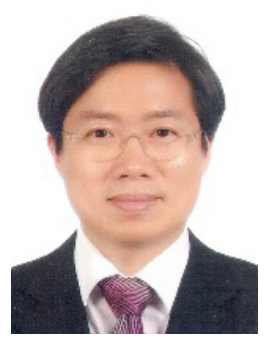

Young-Jae Ryoo received his Ph.D., M.Sc. and B.Sc. degree in the Department of Electrical Engineering, Chonnam National University, South Korea in 1998, 1993 and 1991, respectively. He was a Visiting Researcher in North Carolina A\&T State University, US in 1999. He was a Visiting Professor in the Department of Mechanical Engineering, Virginia Tech, US from 2010-2012. He is currently a Professor in the Department of Control Engineering and Robotics, Mokpo National University, South Korea from 2000. He also served as a Director with the Intelligent Space Laboratory in Mokpo National University, where he is responsible for the research projects in the area of intelligence, robotics and vehicles. He is currently a Board Member of Korean Institute of Intelligent Systems from 2002, an Editor for the Journal of Korean Institute of Electrical Engineering from 2010, an Editor for the Journal of Fuzzy Logic and Intelligent Systems from 2009, and a Committee Member of the International Symposium on Advanced Intelligent Systems from 2005. He served as a General Chair of International Symposium on Advanced Intelligent System in 2014 and 2015. He won the outstanding paper awards, the best presentation awards, and the recognition awards in International Symposiums on Advanced Intelligent Systems. He is the Author of over 200 technical publications. His research interests include intelligent space, Fumanoid robotics, legged robotics, autonomous vehicles, unmanned vehicles, wheeled robotics and biomimetic robotics. 